\title{
Ionization of Hydrogenic 3d State by Electron Impact
}

\author{
Sushmita Banerjee*, Sunil Dhar \\ Department of Mathematics, Chittagong University of Engineering and Technology, Chittagong, Bangladesh \\ Email: *sushmita@cuet.ac.bd
}

How to cite this paper: Banerjee, $S$. and Dhar, S. (2019) Ionization of Hydrogenic $3 \mathrm{~d}$ State by Electron Impact. Open Journal of Microphysics, 9, 29-39.

https://doi.org/10.4236/ojm.2019.94004

Received: August 31, 2019

Accepted: November 2, 2019

Published: November 5, 2019

Copyright (C) 2019 by author(s) and Scientific Research Publishing Inc. This work is licensed under the Creative Commons Attribution International License (CC BY 4.0).

http://creativecommons.org/licenses/by/4.0/

\begin{abstract}
The triple differential cross sections estimation have been ascertained theoretically for the ionization of metastable $3 \mathrm{~d}$-state hydrogen atoms by electron at $150 \mathrm{eV}, 200 \mathrm{eV}$ and $250 \mathrm{eV}$ for assorted kinematic conditions exerting a multiplex scattering principle. The existent new-findings are assimilated with the theoretic data of hydrogenic metastable $2 \mathrm{~S}, 3 \mathrm{~S}$ and $3 \mathrm{P}$ states as well as the hydrogenic ground state experiment. An analysis of the attained observations reveals qualitative fitness with those of compared results specially with hydrogenic ground state BBK model, ground state experimental data and metastable state $2 \mathrm{~S}$ state results. This is a new theoretical work on hydrogenic metastable $3 \mathrm{~d}$ state ionization by electron. The substantial progress of the current study offers a wide scope for empirical study in ionization process.
\end{abstract}

\section{Keywords}

Electron, Ionization, Cross-Section, Scattering

\section{Introduction}

In high energy ion-atom collisions, ionization is one of the most momentous reactions. Electron impact ionization by charged particles is used in solving problems in isolated range of field like astrophysics, plasma physics, fusion technology, radiation physics, etc. The triple differential cross-sections have been attained in ejected electron energy and ejected angles in the electron hydrogen mechanism. The major challenge in the field of electron impact ionization is to develop a general theoretical framework, which will provide an accurate ionization cross sections for many atoms over a relevant impact energy range. Due to its perplexity, the fully quantum mechanical conduct of atomic ionization by electron is possible for the artless cases of hydrogen atom. In this work, atomic hydrogen is used as target in order to focus attention on the colli- 
sion mechanism and to investigate the range of validity of various theoretical models.

Ionization by fast particle was first initiated quantum mechanically by Bethe [1]. Electron atom ionization collision on different cross sections has become gradually interesting over the last four decades both theoretically and experimentally for non-relativistic [2]-[21] as well as relativistic [22]-[25] energies. Various theoretical models applying different kinematical conditions have been vastly used for experimental measurements. Ehrhardt et al. [3] have investigated this more successfully for different energies of the incident electrons from very low to high energies. The present new theoretical study on hydrogenic $3 \mathrm{~d}$ state ionization by electron offers an immense opportunity for further experimental study for ionization of hydrogenic metastable $3 \mathrm{~d}$ state by electron.

Ionization of the hydrogenenic atom by electron is a good image for perturbation theory because of the existence of empirical consequence. In this text, the electron-electron coincidence experiments called (e, 2e) experiments which offer a translucent idea of the kinematics of the collisions by delivering knowledge about the direction of the scattered and ejected electrons. The quantity measured in this kind of experiment is proportional to the TDCS, which represents the angular distribution of the ejected electron for selected incident and scattered electron momenta.

A multiple scattering wave function has been designed for two electrons moving in a coulomb field which include higher order and correlation effects. Lewis integral [26] has been used in the present study for analytic estimation. A multiple scattering principle [5] has been followed in the present study by $150 \mathrm{eV}$, $200 \mathrm{eV}$ and $250 \mathrm{eV}$ energies. The multiple scattering principle [5] plays a vital role in the study of hydrogenic ionization for ground state [14] and metastable states [15] and [18]-[21]. So, the present results seem to be interesting. The existent novel theoretical results make a new dimension on ionization of hydrogenic metastable states. Current results are compared with previous related theories [15], [19], [20].

\section{Theory}

T-matrix element for ionization of hydrogen atoms by electrons pursuing Das and Seal [5] may be taken as

$$
T_{f i}=\left\langle\Psi_{f}^{(-)}\left(\bar{r}_{1}, \bar{r}_{2}\right)\left|V_{i}\left(\bar{r}_{1}, \bar{r}_{2}\right)\right| \Phi_{i}\left(\bar{r}_{1}, \bar{r}_{2}\right)\right\rangle
$$

Here, $\bar{r}_{1}$ and $\bar{r}$ represent the coordinates of the atomic active electron and the incident electron, $\left(\bar{p}_{1}, \bar{p}_{2}\right)$ and $\left(E_{1}, E_{2}\right)$ represent the momenta and energies of the two electrons in the final state and $\left(\bar{p}_{i}, E_{i}\right)$ are the momentum and the energy of the incident electron.

Where the perturbation potential $V_{i}\left(\bar{r}_{1}, \bar{r}_{2}\right)$ is given by

$$
V_{i}\left(\bar{r}_{1}, \bar{r}_{2}\right)=\frac{1}{r_{12}}-\frac{Z}{r_{2}}
$$


The nuclear charge of the hydrogen atom is $Z=1, r_{1}$ and $r_{2}$ are the distance of the two electrons from the nucleus and $r_{12}$ is the distance between two electrons.

The initial channel unperturbed wave function is given in the following form

$$
\Phi_{i}\left(\bar{r}_{1}, \bar{r}_{2}\right)=\frac{\mathrm{e}^{\bar{p}_{i} \cdot \overline{T_{2}}}}{(2 \pi)^{3 / 2}} \phi_{3 d}\left(\bar{r}_{1}\right) .
$$

where

$$
\phi_{3 d}\left(\bar{r}_{1}\right)=\frac{1}{81 \sqrt{6 \pi}}\left(r_{1}^{2}\right)\left(3 \cos ^{2} \theta-1\right) \mathrm{e}^{-r_{1} / 3} .
$$

Here $\lambda_{1}=\frac{1}{3}, \phi_{3 d}\left(\bar{r}_{1}\right)$ is the hydrogenic $3 \mathrm{~d}$-state wave function and $\Psi_{f}^{(-)}\left(\bar{r}_{1}, \bar{r}_{2}\right)$ is approximate wave function is given by [5]

$$
\begin{aligned}
\Psi_{f}^{(-)}\left(\bar{r}_{1}, \bar{r}_{2}\right)= & N\left(\bar{p}_{1}, \bar{p}_{2}\right)\left[\phi_{\bar{p}_{1}}^{(-)}\left(\bar{r}_{1}\right) \mathrm{e}^{\overline{\bar{p}_{2}} \cdot \bar{r}_{2}}+\phi_{\bar{p}_{2}}^{(-)}\left(\bar{r}_{2}\right) \mathrm{e}^{\overline{\bar{p}_{1}} \cdot \bar{r}_{1}}\right. \\
& \left.+\phi_{\bar{p}}^{(-)}(\bar{r}) \mathrm{e}^{i \bar{P} \cdot \bar{R}}-2 \mathrm{e}^{i \bar{p}_{1} \cdot \overline{\bar{p}_{1}}+\overline{p_{2}} \cdot \bar{r}_{2}}\right] /(2 \pi)^{3}
\end{aligned}
$$

where

$$
\begin{gathered}
\bar{r}=\frac{\bar{r}_{2}-\bar{r}_{1}}{2}, \quad \bar{R}=\frac{\bar{r}_{1}+\bar{r}_{2}}{2}, \\
\bar{p}=\left(\bar{p}_{2}-\bar{p}_{1}\right), \quad \bar{P}=\bar{p}_{2}+\bar{p}_{1},
\end{gathered}
$$

The normalization constant $N\left(\bar{p}_{1}, \bar{p}_{2}\right)$ is calculated using Das and Seal [5] and Dhar and Nahar [18].

The Coulomb wave function $\phi_{q}^{(-)}(\bar{r})$ is used from Das and Seal [5] and Dhar and Nahar [18].

The triple differential cross-sections for direct T-Matrix element is given by

$$
\frac{\mathrm{d}^{3} \sigma}{\mathrm{d} \Omega_{1} \mathrm{~d} \Omega_{2} \mathrm{~d} E_{1}}=\frac{p_{1} p_{2}}{p_{i}}\left|T_{f i}\right|^{2}
$$

Here the direct scattering amplitude $f\left(\bar{p}_{1}, \bar{p}_{2}\right)$ is computed in [21].

The exchange scattering amplitude $g\left(\bar{p}_{1}, \bar{p}_{2}\right)$ for hydrogen atom are

$$
\begin{aligned}
& f\left(\bar{p}_{1}, \bar{p}_{2}\right)=-(2 \pi)^{2} T_{f i} \\
& g\left(\bar{p}_{1}, \bar{p}_{2}\right)=f\left(\bar{p}_{2}, \bar{p}_{1}\right) .
\end{aligned}
$$

TDCS with exchange effects is given by

$$
\frac{\mathrm{d}^{3} \sigma}{\mathrm{d} \Omega_{1} \mathrm{~d} \Omega_{2} \mathrm{~d} E_{1}}=\frac{p_{1} p_{2}}{p_{i}}\left[\frac{3}{4}|f-g|^{2}+\frac{1}{4}|f+g|^{2}\right] .
$$

Right hand side of Equation (3) is computed numerically by exerting computer programming language Mat-Lab.

\section{Results and Discussions}

Ionization of hydrogenic metastable $3 \mathrm{~d}$ state by electron impact is presented for 
different kinematic conditions. The existent new results are assimilated with the hydrogenic ground state theoretical results [10], [13] and the absolute data [3]. The ionization results of hydrogenic metastable $2 S$ state [15], 3S state [19] and $3 \mathrm{P}$ state [20] are also covered here for comparison with our new theoretical study results. In this text, the ejected angle $\theta_{1}$ varies from $0^{\circ}$ to $360^{\circ}$ where scattering angles $\theta_{2}$ is fixed. The incident electron energy $E_{i}=150 \mathrm{eV}, 200 \mathrm{eV}$ and $250 \mathrm{eV}$ are taken here. In all diagram, $\theta_{1}\left(0^{\circ}-180^{\circ}\right)$ and $\phi=0^{\circ}$ is indicated as recoil field while $\theta_{1}\left(180^{\circ}-360^{\circ}\right)$ and $\phi=180^{\circ}$ is referred as binary field.

The obtained results are sketched corresponding to the different scattering angles $\theta_{2}=3^{\circ}$ Figure 1 (a) for ejected electron energies $E_{1}=5 \mathrm{eV}$ as well as $15^{\circ}$ Figure $1(\mathrm{~b}), 25^{\circ}$ Figure 1 (c) for $E_{1}=50 \mathrm{eV}$ considering the ejected angle $\theta_{1}$ from $30^{\circ}$ to $100^{\circ}$. Also the existent results are plotted for the scattering angle $\theta_{2}=5^{\circ}$ Figure 2(a), $7^{\circ}$ Figure 2(b), $9^{\circ}$ Figure 2(c), $11^{\circ}$ Figure $2(\mathrm{~d}), 15^{\circ}$ Figure 2(e), $20^{\circ}$ Figure 2(f). Again, present results are plotted for incident electron energy $E_{i}=150 \mathrm{eV}$ and ejected electron energy $E_{1}=5 \mathrm{eV}$ for $\theta_{2}=7^{\circ}$ Figure 3(a), $\theta_{2}=9^{\circ}$ Figure 3(b), $\theta_{2}=11^{\circ}$ Figure 3(c) as well as sketched for incident electron energy $E_{i}=200 \mathrm{eV}$ and ejected electron energy $E_{1}=5 \mathrm{eV}$ for $\theta_{2}=5^{\circ}$ Figure 4(a), $\theta_{2}=7^{\circ}$ Figure 4(b), $\theta_{2}=9^{\circ}$ Figure 4(c).

In Figure 1(a) peak values of present first Born black dash curve, direct black

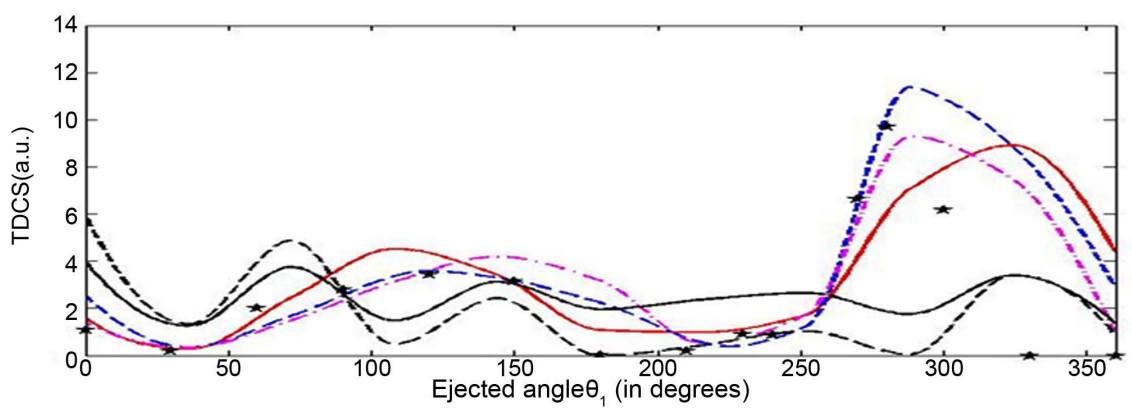

(a)

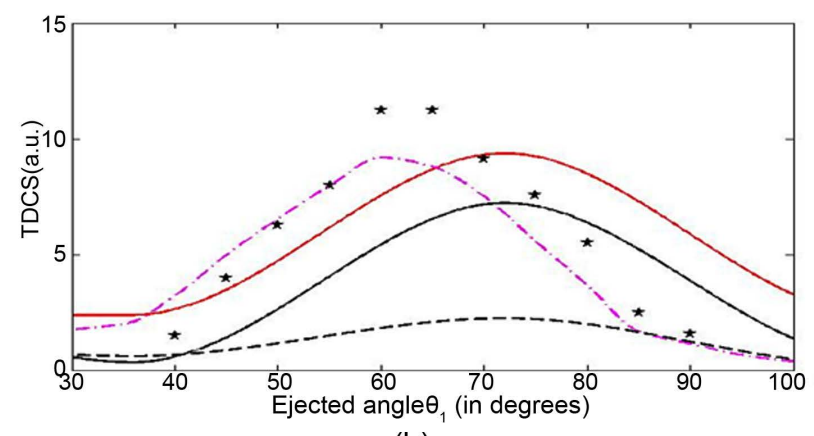

(b)

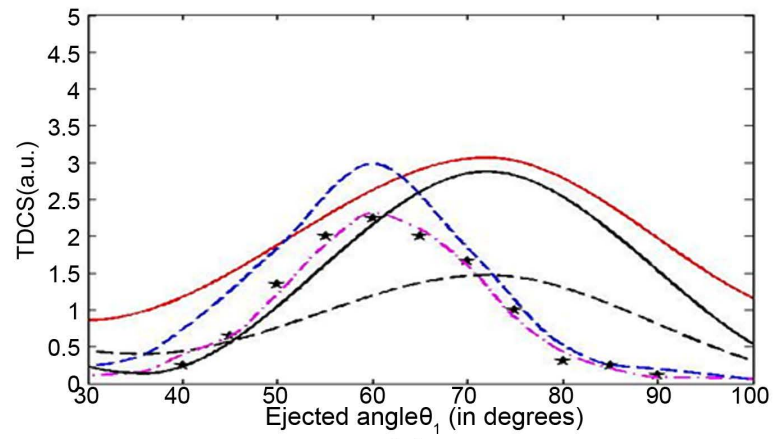

(c)

Figure 1. The TDCS impact as a function of the ejected electron angle $\theta_{1}$ for atomic hydrogen by incident electron energy $E_{i}=250 \mathrm{eV}$ with (a) $E_{1}=5 \mathrm{eV}$ and $\theta_{2}=3^{\circ}$, (b) $E_{1}=50 \mathrm{eV}$ and $\theta_{2}=15^{\circ}$, (c) $E_{1}=50 \mathrm{eV}$ and $\theta_{2}=25^{\circ}$. Theory: Black dash curve, Black continuous curve, Red perfect curve illustrate present first Born, direct and exchange effect results respectively. Also Blue dash curve demonstrate Hydrogenic ground state Second Born results [13], Magenta dash dotted curve reveal Hydrogenic ground state BBK model [10] and Star indicated Hydrogenic ground state experiment [3] (multiplied by 0.00224). 
continuous curve and exchange red perfect curve results show good qualitative agreement with those of the compared results [3], [10], [13] in the recoil field but show somewhat vary in the binary field. This may be arised due to the change of the hydrogenic metastable states ionization. Current first Born, direct and exchange results are slightly shifted right from other compared results in the binary field. Present exchange results are conjoined with hydrogenic ground state experimental result [3] at about $\theta_{1}=40^{\circ}$ and $\theta_{1}=150^{\circ}$ where it is expected.

In Figure 1(b), current first Born result show flat peak magnitude and direct results show minimum peak magnitude than the hydrogenic ground state experimental results [3], hydrogenic ground state BBK model [10] and present exchange result. In the recoil field, present exchange results show uniquely closer with low magnitude to the hydrogenic ground state experimental result [3] but in the binary field, present direct and exchange outcomes are moved forward direction from the compared results [3], [10].

In Figure 1(c), Existence first Born result is assembled with hydrogenic ground state experimental result [3], BBK result [10] and current direct result. The peak pattern of present direct and exchange results provide similar but shifted onward direction from hydrogenic ground state second Born results [13]. Peak magnitude of existence exchange results which is upper than other compared results [3], [10] in both recoil and binary field.

In Figure 2(a), in the recoil field, about $\theta_{1}=60^{\circ}$, present first Born results make a petty peak and on the other side, current direct configuration make a short lobe with 3S-state results [20] and current exchange result. In the binary field, the magnitude of obtained exchange outcome is decreased from other compared results [15], [19], [20].

In Figure 2(b), present first Born curve express identical behavior with 3P-state curve [20] and direct curve depict similar conduct with 3S-state curve [20] in the recoil field. The present exchange result is reversely jointed with $3 \mathrm{P}$-state results [20] at higher ejected angle about $\theta_{1}=288^{\circ}$.

In Figure 2(c), in the recoil field at approximate $\theta_{1}=60^{\circ}$, existent first Born structure create same peak pattern with $2 S$-state structure [15] whereas present direct curve conjoined contrarily with 2S-state [15] and existent exchange curve make a nice lobe. New exchange result form minimum peak magnitude than 2S-state result [15] in the binary field.

In Figure 2(d), current attained first Born configuration conjoined with 2S-state [15] and 3S-state [19] configurations in the recoil field near about $\theta_{1}=45^{\circ}$. Present direct including exchange results make flat form where $2 \mathrm{~S}$-state [15] result create a lower dip at about $\theta_{1}=252^{\circ}$. Present direct and exchange structure give a dull peak where $2 \mathrm{~S}$-state [15] structure shows clear peak at ejected angle about $288^{\circ}$.

In Figure 2(e), existent first Born curve provides almost similar behavior with 3S-state [19], 3P-state [20], present direct and exchange curves but shows a gross difference with the results of $2 \mathrm{~S}$-state [15] in recoil field at about $\theta_{1}=45^{\circ}$. The existent first Born diagram give same peak with 3P-state [20], present direct and 
exchange diagram but $2 \mathrm{~S}$-state [15] diagram reveal small lobe at approximate $252^{\circ}$.

In Figure 2(f), present first born configuration meets contrarily with 3S-state [19] configuration while present direct and present exchange configuration meets reversely with 3P-state [20] configuration in the recoil field at about $\theta_{1}=148^{\circ}$. Present exchange diagram display opposite form with 3P-state [20] diagram in both recoil and binary field.

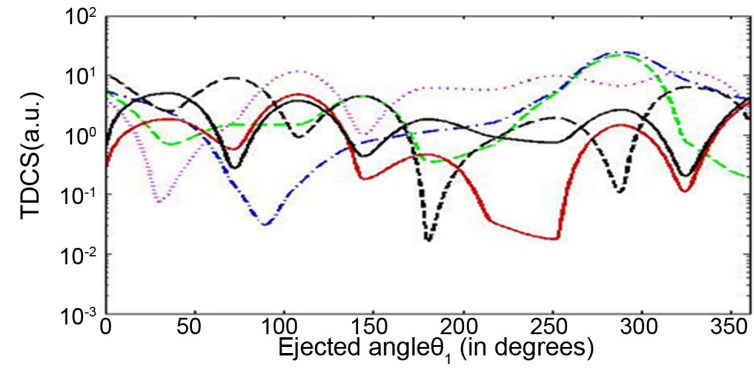

(a)

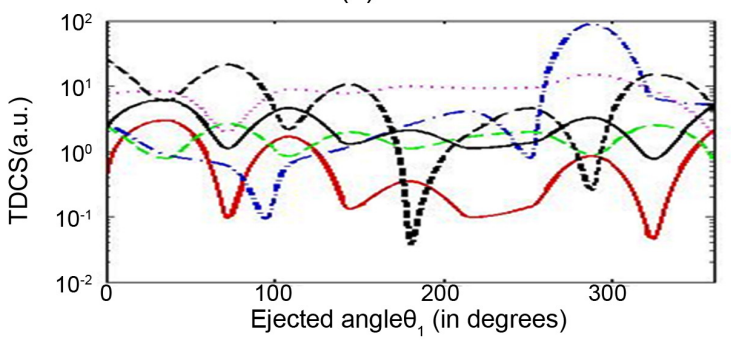

(b)

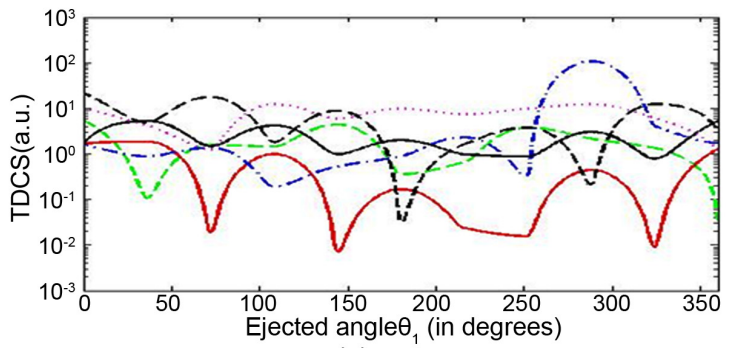

(c)

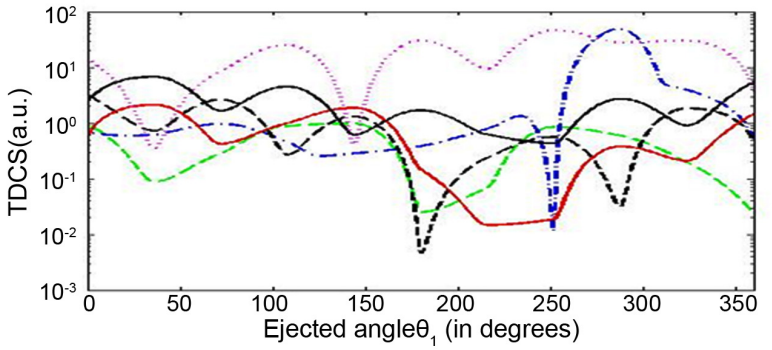

(d)

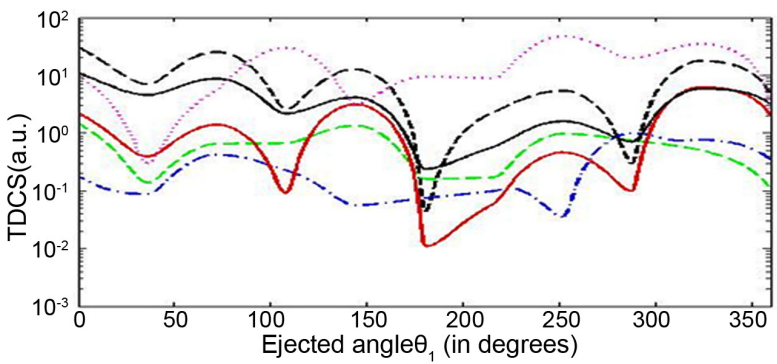

(e)

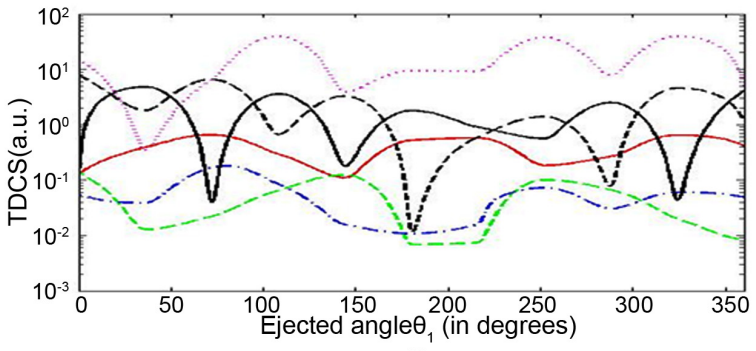

(f)

Figure 2. The TDCS impact as a function of the ejected electron angle $\theta_{1}$ for atomic hydrogen by incident electron energy $E_{i}=250 \mathrm{eV}$ with ejected electron energy $E_{1}=5 \mathrm{eV}$ and (a) $\theta_{2}=5^{\circ}$, (b) $\theta_{2}=7^{\circ}$ (c) $\theta_{2}=9^{\circ}$, (d) $\theta_{2}=11^{\circ}$, (e) $\theta_{2}=15^{\circ}$, (f) $\theta_{2}=20^{\circ}$. Theory: Black dash curve, Black continuous curve, Red perfect curve exhibit present first Born, direct and exchange effect results respectively. Also Green dash dotted curve display hydrogenic 3P-state result [20], Magenta dotted curve expose hydrogenic 3S-state result [19] and Blue dash dotted curve demonstrate hydrogenic 2S-state result [15].

To understand these structures, a table (please see Table 1) which is given for assimilation of hydrogenic ionization of 3d state with 2S-state, 3S-state, 3P-state results.

In Figure 3(a), It is observed that current first Born configuration coincides with present direct and exchange configurations at $\theta_{1}=180^{\circ}$. Present direct curve exhibit similar nature with exchange curve in the recoil field but shows a little bit different nature in the binary field. 
Table 1. Electron impact hydrogenic ionization of 2S-state, 3S-state direct, 3P-state exchange and $3 \mathrm{~d}$ state results are discriminated for $E_{i}=250 \mathrm{eV}, \theta_{2}=15^{\circ}$ and $E_{1}=5 \mathrm{eV}$.

\begin{tabular}{cccccc}
\hline \multirow{2}{*}{ Ejected angle $\left(\theta_{1}\right)$} & $2 S$ & $3 S$ & $3 \mathrm{P}$ & \multicolumn{2}{c}{$3 \mathrm{~d}$} \\
\cline { 5 - 6 } & & & & Direct & Exchange \\
\hline 0 & 0.1823 & 10.0001 & 1.5029 & 11.2090 & 1.7620 \\
76 & 0.0895 & 0.3002 & 0.1405 & 4.6003 & 0.3605 \\
108 & 0.4251 & 5.7523 & 0.6569 & 8.8108 & 1.2505 \\
144 & 0.2301 & 30.2539 & 0.6770 & 2.1945 & 0.0684 \\
180 & 0.0569 & 3.2101 & 1.3530 & 4.1788 & 4.0890 \\
216 & 0.0753 & 9.5999 & 0.1625 & 0.2408 & 0.0099 \\
252 & 0.1001 & 9.0001 & 0.1729 & 0.5900 & 0.0400 \\
288 & 0.0356 & 47.9753 & 0.9793 & 1.6147 & 0.7353 \\
324 & 1.0012 & 20.0010 & 0.7521 & 0.7171 & 0.0486 \\
360 & 0.7723 & 35.2539 & 0.5025 & 5.9222 & 3.5060 \\
\hline
\end{tabular}

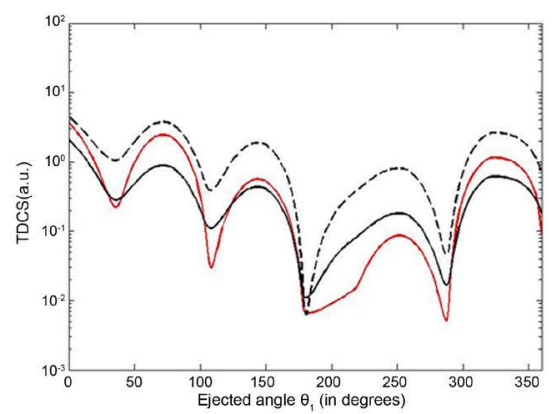

(a)

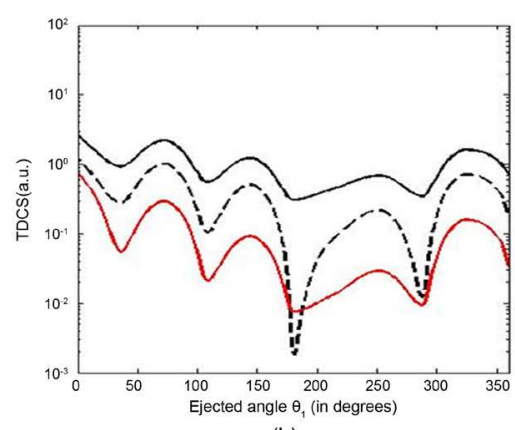

(b)

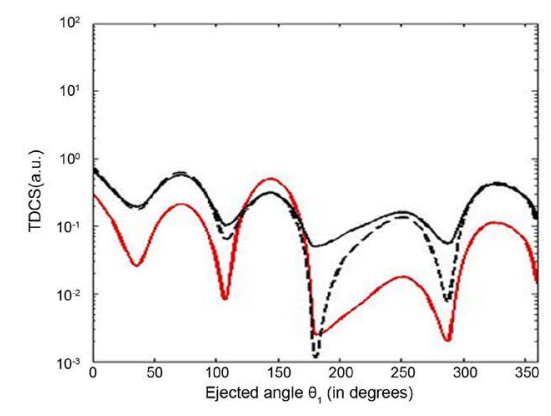

(c)

Figure 3. The TDCS impact as a function of the ejected electron angle $\theta_{1}$ for atomic hydrogen by incident electron energy $E_{i}=150 \mathrm{eV}$ and ejected electron energy $E_{1}=5 \mathrm{eV}$ with (a) $\theta_{2}=7^{\circ}$ (b) $\theta_{2}=9^{\circ}$ (c) $\theta_{2}=11^{\circ}$ Theory: Black dash curve, black continuous curve and red perfect curve expose present first Born, direct \& exchange results respectively.

In Figure 3(b), first Born TDCS curve create a lower dip at about $\theta_{1}=180^{\circ}$ and $\theta_{1}=280^{\circ}$ whereas present direct and exchange results make flat. Direct curve with high magnitude display almost similar pattern with present exchange curve in both recoil and binary field.

In Figure 3(c), The first Born diagram is overlapped with direct and exchange diagrams many times at distinct ejected angle both in recoil and binary field. The first Born and exchange results create two lower dip in the binary field at approximate $\theta_{1}=180^{\circ}$ and $\theta_{1}=280^{\circ}$.

Here a table (please see Table 2) which is presented for assimilation of first Born, direct and exchange results of hydrogenic 3d-state ionization.

In Figure 4(a), current attained exchange configuration conjoined with present direct configurations whereas first Born configurations with high 
Table 2. First Born, direct and exchange results of $\mathrm{H}(3 \mathrm{~d})$ are distinguished for $E_{i}=150 \mathrm{eV}$, $E_{1}=5 \mathrm{eV}$ and $\theta_{2}=9^{\circ}$.

\begin{tabular}{cccc}
\hline \multirow{2}{*}{ Ejected angle $\left(\theta_{1}\right)$} & \multicolumn{3}{c}{$3 \mathrm{~d}$} \\
\cline { 2 - 4 } 0 & First Born $(\mathrm{B} 1)$ & Direct & Exchange \\
\hline 36 & 1.2682 & 2.6889 & 0.7814 \\
72 & 0.2867 & 0.9488 & 0.0557 \\
108 & 1.0354 & 2.2400 & 0.2991 \\
144 & 0.1057 & 0.5639 & 0.0212 \\
180 & 0.5168 & 1.2501 & 0.0936 \\
216 & 0.0017 & 0.3130 & 0.0077 \\
252 & 0.0946 & 0.4644 & 0.0142 \\
288 & 0.2214 & 0.6953 & 0.0297 \\
324 & 0.0122 & 0.3524 & 0.0095 \\
360 & 0.7230 & 1.6426 & 0.1609 \\
& 0.1827 & 0.7289 & 0.0338 \\
\hline
\end{tabular}

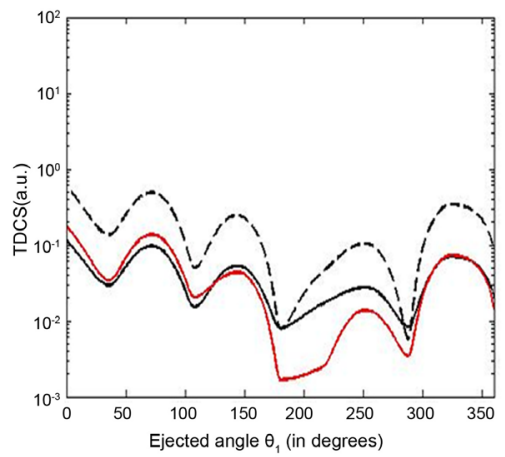

(a)

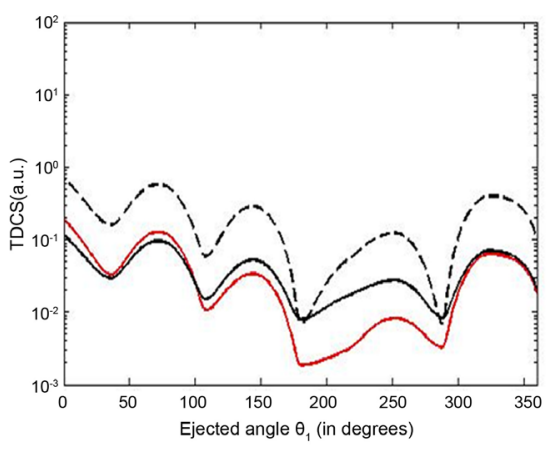

(b)

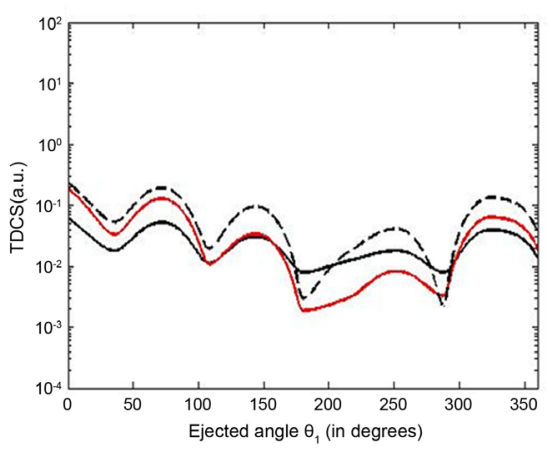

(c)

Figure 4. The TDCS impact as a function of the ejected electron angle $\theta_{1}$ for atomic hydrogen by incident electron energy $E_{i}=200 \mathrm{eV}$ and ejected electron energy $E_{1}=5 \mathrm{eV}$ with (a) $\theta_{2}=5^{\circ}$ (b) $\theta_{2}=7^{\circ}$ (c) $\theta_{2}=9^{\circ}$ Theory: Black dash curve, black continuous curve and red perfect curve expose present first Born, direct \& exchange results respectively.

magnitude display almost similar pattern with present exchange configurations in recoil field. Exchange results make flat with Present direct curve but first Born curve create a dull lobe at about $\theta_{1}=180^{\circ}$ in the binary field.

In Figure 4(b), The existent exchange diagram give same peak with existent direct diagram in the recoil field but first Born diagram reveal gross difference with direct and exchange diagram both in recoil and binary field. At about $\theta_{1}=280^{\circ}$, existent exchange and direct diagram create short lobe but first Born result make a long lobe.

In Figure 4(c), The first Born diagram is overlapped with direct and exchange diagrams many times at distinct ejected angle both in recoil and binary field. The first Born and exchange results create two lower dip in the binary field at approximate $\theta_{1}=180^{\circ}$ and $\theta_{1}=280^{\circ}$. 


\section{Conclusion}

The present calculation exposes additional possible structure of the cross-section curves for intermediate momentum transfer in the hydrogenic 3d-state ionization at $150 \mathrm{eV}, 200 \mathrm{eV}$ and $250 \mathrm{eV}$ impact energy. In the present estimation, the correlated three particle final state wave function of Das and Seal [5] has been followed. New theoretical computational observations for ionization of hydrogenic $3 \mathrm{~d}$ state by electrons may be generalized for application to the other atom as well as ions and which may play a vital role to provide much interesting and potential results in this field of research.

\section{Acknowledgements}

The computational works are executed in the Simulation Lab of Department of Mathematics, Chittagong University of Engineering and Technology, Chittagong-4349, Bangladesh.

\section{Conflicts of Interest}

The authors declare no conflicts of interest regarding the publication of this paper.

\section{References}

[1] Bethe, H.Z. (1930) Theorie des Durchgangs schneller Korpuskularstrahlen durch Materie. Annalen der Physik, 397, 325-400. https://doi.org/10.1002/andp.19303970303

[2] Ehrhardt, H., Knoth, G., Schlemmer, P. and Jung, K. (1985) Absolute H(e, 2e)p Cross Section Measurements: Comparison with First and Second Order. Physics Letters A, 110, 92-94. https://doi.org/10.1016/0375-9601(85)90326-3

[3] Ehrhardt, H., Knoth, G., Schlemmer, P. and Jung, K. (1986) Differential Cross Sections of Direct Single Electron Impact Ionization. Zeitschrift für Physik D Atoms, Molecules and Clusters, 1, 3-32. https://doi.org/10.1007/BF01384654

[4] Das, J.N. (1990) Momentum-Space Analysis of Scattering States with Possible Application to Atomic Ionization. Physical Review A, 42, 1376-1378. https://doi.org/10.1103/PhysRevA.42.1376

[5] Das, J.N. and Seal, S. (1993) Electron-Hydrogen Atom Ionization Collisions at Intermediate (510-2010) and High (> 2010) Energies. Physical Review A, 47, 29782986. https://doi.org/10.1103/PhysRevA.47.2978

[6] Das, J.N. and Dhar, S. (1999) Energy Spectrum of Ejected Electrons in Ionization of Hydrogen Atoms by Electrons. Pramana, 53, 869-875. https://doi.org/10.1007/s12043-999-0121-9

[7] Byron, F.W., Joachen, C.J. and Piraux, B. (1980) Triple Differential Cross Sections for the Ionisation of Atomic Hydrogen by Fast Electrons: A Second Born Treatment. Journal of Physics B: Atomic, Molecule and Optical Physic, 13, L673. https://doi.org/10.1088/0022-3700/13/22/005

[8] Byron, F.W., Joachain, C.J. and Piraux, B. (1986) Theory of Coplanar Asymmetric (e, 2e) Reactions in Helium. Journal of Physics B: Atomic, Molecular and Optical Physics, 19, 120. https://doi.org/10.1088/0022-3700/19/8/015 
[9] Brauner, M., Briggs, M. and Klar, H. (1991) Structures in Differential Cross Sections for Positron Impact Ionization of Hydrogen. Journal of Physics B: Atomic, Molecular and Optical Physics, 24, 2227. https://doi.org/10.1088/0953-4075/24/8/030

[10] Brauner, M., Briggs, J.S. and Klar, H. (1989) Triply-Differential Cross Sections for Ionisation of Hydrogen Atoms by Electrons and Positrons. Journal of Physics B: Atomic, Molecular and Optical Physics, 22, 2265-2287.

https://doi.org/10.1088/0953-4075/22/14/010

[11] Berakder, J. and Klar, H. (1993) Structures in Triply and Doubly Differential Ionization Cross Sections of Atomic Hydrogen. Journal of Physics B: Atomic, Molecular and Optical Physics, 26, 3891. https://doi.org/10.1088/0953-4075/26/21/023

[12] Berakdar, J., Engelns, A. and Klar, H. (1996) Oriented and Aligned Two Electron Continue. Journal of Physics B: Atomic, Molecular and Optical Physics, 29, 1109. https://doi.org/10.1088/0953-4075/29/5/019

[13] Dal Capppello, C., Haddadou, A., Menas, F. and Roy, A.C. (2011) The Second Born Approximation for the Single and Double Ionization of Atoms by Electrons and Positrons. Journal of Physics B: Atomic, Molecular and Optical Physics, 44, Article ID: 015204. https://doi.org/10.1088/0953-4075/44/1/015204

[14] Das, J.N. and Dhar, S. (1996) Symmetric Scattering in Electron and Positron Impact Ionization of Metastable 2S-State Hydrogen Atom. Pramana-Journal of Physics, 47, 263-269. https://doi.org/10.1007/BF02848527

[15] Dhar, S. (1996) Electron Impact Ionisation of Metastable 2S-State Hydrogen Atoms. Australian Journal of Physics, 49, 937-944. https://doi.org/10.1071/PH960937

[16] Vučič, S., Potvliege, R.M. and Joachain, C.J. (1987) Second Born Triple-Differential Cross Sections for the Coplanar Asymmetric Ionization of $\mathrm{H}(2 \mathrm{~S})$ by Fast Electrons. Physical Review A, 35, 1446-1449. https://doi.org/10.1103/PhysRevA.35.1446

[17] Qi, Y.Y., Ning, L.N., Wang, J.G. and Qu, Y.Z. (2013) Plasma Effect on Fast-Electron-Impact Ionization from 2P State of Hydrogen-Like Ions. Physics of Plasmas, 20, Article ID: 123301. https://doi.org/10.1063/1.4833616

[18] Dhar, S. and Nahar, N. (2016) Triple Differential Cross-Sections for the Ionization of Metastable 2P-State Hydrogen Atoms by Electrons with Exchange Effects. Pramana-Journal of Physics, 87, 69. https://doi.org/10.1007/s12043-016-1281-z

[19] Noor, T. and Dhar, S. (2017) The Triple Differential Cross Sections for Electron Impact Ionization of Metastable 3S State Hydrogen Atoms with Exchange Effect. Open Journal of Microphysics, 7, 53-65. https://doi.org/10.4236/ojm.2017.73004

[20] Akter, S., Dhar, S., Nahar, N. and Das, L.C. (2018) Ionization of Metastable 3P State Hydrogen Atom by Electron with Exchange Effects. Pramana Journal of Physics, 91, 78. https://doi.org/10.1007/s12043-018-1648-4

[21] Banerjee, S., Dhar, S. and Hoque, A. (2018) Triple Differential Cross-Sections for Ionization of H(3d) by Incident Electron. Open Journal of Microphysics, 8, 30-41. https://doi.org/10.4236/ojm.2018.84005

[22] Das, J.N. and Chakraborty, K. (1985) Atomic Inner-Shell Ionization. Physical Review $A$, 32, 176-180. https://doi.org/10.1103/PhysRevA.32.176

[23] Das, J.N. and Dhar, S. (1998) Calculation of Triple Differential Cross-Sections of K-Shell Ionization of Medium-Heavy Atoms by Electrons for Symmetric Geometry. Pramana, 51, 751-756. https://doi.org/10.1007/BF02832607

[24] Das, J.N. and Dhar, S. (1998) Energy Spectrum of Scattered Electrons in K-Shell Ionization of Medium to Heavy Atoms by Relativistic Electrons. Journal of Physics B: Atomic, Molecular and Optical Physics, 31, 2355. 
https://doi.org/10.1088/0953-4075/31/10/021

[25] Dhar, S. (2008) The Energy Spectrum of Scattered Particles in the K-Shell Ionization of Medium Heavy Atoms by Relativistic Electrons and Positrons with Exchange Effects. Journal of Physics B: Atomic and Molecular Physics, 41, Article ID: 155204. https://doi.org/10.1088/0953-4075/41/15/155204

[26] Lewis, R.R. (1956) Potential Scattering of High-Energy Electrons in Second Born Approximation. Physical Review, 102, 537-543.

https://doi.org/10.1103/PhysRev.102.537 